\title{
Seeing green... augmentation of lymph node assessment with near-infrared imaging in esophageal cancer resections
}

\author{
Inderpal S. Sarkaria, MD, and James D. Luketich, MD
}

\footnotetext{
From the Division of Thoracic and Foregut Surgery, Department of Cardiothoracic Surgery, University of Pittsburgh Medical Center, Pittsburgh, Pa.

Disclosures: Authors have nothing to disclose with regard to commercial support.

Received for publication May 6, 2016; accepted for publication May 6, 2016

Address for reprints: James D. Luketich, MD, Department of Cardiothoracic Surgery, University of Pittsburgh

Medical Center, 200 Lothrop St, Suite C-800, Pittsburgh, PA 15213 (E-mail: luketichjd@upmc.edu).

J Thorac Cardiovasc Surg 2016;152:555-6

$0022-5223 / \$ 36.00$

Copyright (C) 2016 Published by Elsevier Inc. on behalf of The American Association for Thoracic Surgery

http://dx.doi.org/10.1016/j.jtcvs.2016.05.015
}

Visual and tactile senses remain the primary tools of the modern surgeon. For the cancer surgeon, the ability to assess the scope of neoplastic involvement, including the margin and metastatic involvement of tumor, can be limited by these basic modalities. Although advances in preoperative imaging have improved the physician's ability to judge the scope of disease, few, if any, have provided meaningful intraoperative visual means to aid decision making in "real time." In addition, the increasing focus on advanced minimally invasive techniques has placed a greater priority on visual cues as the primary means of informative feedback to the surgeon. Emerging imaging technologies may hold the promise of improving accurate visual perception during the conduct of surgery. Near-infrared imaging (NIFI) has emerged as one such promising technology during surgery in several solid tumors, including esophagogastric neoplasms. $^{1-3}$

In this prospective study, Dr Hachey and colleagues ${ }^{4}$ describe the first reported experience with NIFI using indocyanine green (ICG) in the intraoperative assessment of local and regional lymph nodes during minimally invasive esophagectomy for esophageal cancer. With peritumoral ICG injection, NIFI was successful in identifying regional lymph nodes in 6 of the 9 study patients and in all 4 patients in whom ICG was combined with human serum albumin as the solvent. As per the primary aim of the study, the authors demonstrated the safety and feasibility of NIFI in this patient cohort. As already expertly discussed by the authors, the study further asks whether technologies such as NIFI using ICG improve staging independently of, or due to, better informing the extent of lymphadenectomy or identification of additional metastatic disease. Accordingly, can they affect the selection of initial or adjuvant clinical therapies and ultimately improve clinical outcomes?

The ability of NIFI with ICG to identify additional regional lymph nodes in this study, with or without induction therapies, is encouraging. Although none of the NIFI-positive lymph nodes harbored histologic gross or

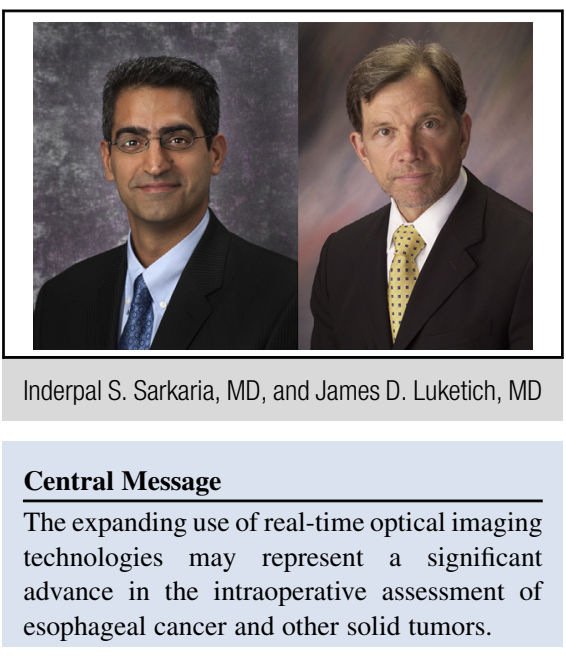

See Article page 546.

micrometastatic disease, the ability to augment total lymph node counts alone may be of benefit given the potential survival advantage with the increased number of nodes harvested. ${ }^{5}$ Whether, as the authors also suggest, NIFI with ICG nodal staging may inform decisions regarding endoscopic versus surgical resection in early-stage T1 tumors will require far greater certainty regarding the sensitivity and specificity of NIFI to confidently rule out occult nodal metastasis. Although caution clearly must be exercised in extrapolating the clinical significance of the authors' findings in this small safety and feasibility study, the implications of real-time lymph node assessment in these operations are significant.

From a broader perspective, this study represents a burgeoning field of study in optical surgical imaging, inclusive of NIFI-based methods for cancer. ${ }^{6}$ Several NIFI-based technologies are currently in preclinical or clinical development, and include the use of an increasing range of tumor or tissue specific fluorophores beyond ICG. ${ }^{7}$ As indicated by the authors, these technologies hold significant promise in providing powerful tools to enhance surgeons' real-time, intraoperative visual perception of cancer biology during the conduct of surgery. Although the current study represents an early investigation with limited immediate applicability in esophageal lymph node assessment, it is of significant importance and, in our opinion, represents a promising seminal investigation in the applicability of NIFI technology in esophageal cancer operations. 


\section{References}

1. Chi C, Du Y, Ye J, Kou D, Qui J, Wang J, et al. Intraoperative imaging-guided cancer surgery: from current fluorescence molecular imaging methods to future multi-modality imaging technology. Theranostics. 2014;4:1072-84.

2. Sarkaria IS, Bains MS, Finley DJ, Adusumilli PS, Huang J, Rusch VW, et al Intraoperative near-infrared fluorescence imaging as an adjunct to roboticassisted minimally invasive esophagectomy. Innovations (Phila). 2014;9:391-3.

3. Herrera-Almario G, Patane M, Sarkaria I, Strong VE. Initial report of near-infrared fluorescence imaging as an intraoperative adjunct for lymph node harvesting during robot-assisted laparoscopic gastrectomy. J Surg Oncol. 2016;113:768-70.
4. Hachey KJ, Gilmore DM, Armstrong KW, Harris SE, Hornick JL, Colson YL, Wee JO. Safety and feasibility of near-infrared image-guided lymphatic mapping of regional lymph nodes in esophageal cancer. J Thorac Cardiovasc Surg. 2016;152:546-54.

5. Rizk NP, Ishwaran H, Rice TW, Chen LQ, Schipper PH, Kessler KA, et al. Optimum lymphadenectomy for esophageal cancer. Ann Surg. 2010;251:46-50.

6. Vahrmeijer AL, Hutteman M, van der Vorst JR, van de Velde CJ, Frangioni JV. Image-guided cancer surgery using near-infrared fluorescence. Nat Rev Clin Oncol. 2013;10:507-18.

7. Garland M, Yim JJ, Bogyo M. A bright future for precision medicine: advances in fluorescent chemical probe design and their clinical application. Cell Chem Biol. 2016;23:122-36. 